\title{
Transposition
}

Musique et Sciences Sociales

$8 \mid 2019$

Musique : patrimoine immatériel ?

\section{Aaron S. Allen \& Kevin Dawe (eds.), Current Directions in Ecomusicology: Music, Culture, Nature}

New York, Routledge, 2017

\section{William Tallotte}

\section{(2) OpenEdition}

\section{Journals}

Édition électronique

URL : http://journals.openedition.org/transposition/3077

DOI : 10.4000/transposition.3077

ISSN : 2110-6134

Éditeur

CRAL - Centre de recherche sur les arts et le langage

Référence électronique

William Tallotte, « Aaron S. Allen \& Kevin Dawe (eds.), Current Directions in Ecomusicology: Music, Culture, Nature », Transposition [En ligne], 8|2019, mis en ligne le 15 septembre 2019, consulté le 17 décembre 2020. URL : http://journals.openedition.org/transposition/3077 ; DOI : https://doi.org/ 10.4000/transposition.3077

Ce document a été généré automatiquement le 17 décembre 2020.

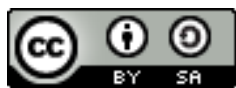

La revue Transposition est mise à disposition selon les termes de la Licence Creative Commons Attribution - Partage dans les Mêmes Conditions 4.0 International. 


\section{Aaron S. Allen \& Kevin Dawe (eds.), Current Directions in Ecomusicology: Music, Culture, Nature}

New York, Routledge, 2017

William Tallotte

\section{RÉFÉRENCE}

Aaron S. Allen \& Kevin Dawe (eds.), Current Directions in Ecomusicology: Music, Culture, Nature, New York, Routledge, 2017, 314 p.

1 Cet ouvrage collectif, coordonné et édité par le musicologue américain Aaron S. Allen et l'ethnomusicologue britannique Kevin Dawe, s'inscrit dans la continuité des travaux qui, depuis la fin des années 2000, tentent de fédérer autour d'un terme nouveau, " ecomusicology », l'ensemble des recherches afférentes aux relations entre musique et environnement ${ }^{1}$. Les auteurs présentent ici vingt textes inédits, particulièrement diversifiés, et précisent, de façon critique et argumentée, ce qu'est, ou du moins pourrait être, l'écomusicologie.

2 Dans leur introduction (chap. 1), Allen et Dawe définissent l'écomusicologie comme l'étude des relations ou intersections possibles entre musique/son, culture/société et nature/environnement (p. 2, 4). De prime abord, donc, l'écomusicologie serait une extension de l'ethnomusicologie. Rien de moins sûr pourtant. On remarque en effet que le couple culture/société disparaît régulièrement du texte; on lit par exemple (p.1): "Ecomusicology considers musical and sonic issues, both textual and performative, related to ecology and the natural environment ». De là, deux interrogations : doit-on estimer 1) que cette disparition n'en est pas une dans la mesure où le préfixe "éco " (du grec oikos, la "maisonnée» comme lieu d'habitation et de production) induit toujours et partout les notions de culture et de société ? Ou 2) que l'écomusicologie se focalise en premier lieu sur les rapports entre son et environnement et ne place pas 
nécessairement l'homme, ni peut-être même la musique, au centre de son projet? Allen et Dawe n'entrent pas dans ce débat. Ils considèrent simplement l'écomusicologie comme un champ thématique, un lieu de rencontre ouvert, décloisonné, dans lequel toute recherche sur le son et/ou la musique peut entrer en dialogue avec une ou plusieurs formes d'écologies - de l'écologie scientifique à l'écologie politique, en passant par l'écocritique. Il n'y aurait donc pas une écomusicologie, soit une discipline qu'il faudrait à tout prix définir et délimiter, mais plutôt des écomusicologies, soit un ensemble de pratiques mêlant à souhait une multitude d'intérêts et de méthodes.

3 L'ouvrage est composé de dix-neuf chapitres, relativement courts, écrits seuls ou à deux, par sept musicologues (Allen, Ryan, Sonevytsky, Stimeling, Drott, Feisst, Von Glahn), sept ethnomusicologues (Dawe, Waterman, Post, Titon, Seeger, Simonett, Edwards), deux écologues (Boyle, Guyette), deux spécialistes en études environnementales (Mark, Ivakhiv), un psychologue de la musique (Windsor), une historienne des sciences (Hui), un anthropologue de la communication (Pedelty) et un spécialiste des médias (Ingram). Les chapitres sont regroupés en quatre grandes parties, chaque fois précédées d'une brève introduction: "Ecological directions " (chap. 2-5), « Fieldwork directions » (chap. 6-10), «Critical directions » (chap. 11-15) et «Textual directions » (chap. 16-20). Bien que cette division semble un peu artificielle la plupart des chapitres pouvant très bien s'inscrire dans au moins deux parties -, on notera toutefois une trajectoire assez logique allant grosso modo des études les plus empiriques aux études les plus interprétatives. D'un point de vue formel, l'ouvrage est très bien conçu. On appréciera en particulier la présence d'un index général, d'un excellent glossaire, fort de 62 entrées, et d'un site web (http:// www.ecomusicology.info/cde) donnant accès à des documents additionnels : bibliographies, diagrammes, tableaux, vidéos.

W. Alice Boyle et Ellen Waterman (chap. 2) ouvrent le débat et la première partie de l'ouvrage. Elles comparent les méthodologies de la bioacoustique aviaire et de l'ethnomusicologie afin de comprendre ce qu'une méthode de type scientifique, croisant l'observation, la comparaison et l'expérimentation, pourrait apporter à une analyse de la performance musicale. Dans le chapitre suivant, Margaret Q. Guyette et Jennifer $\mathrm{C}$. Post explorent à leur tour la possibilité d'une écomusicologie où sciences de la nature et sciences humaines se croisent, se complètent, se renforcent. À travers deux études de cas, elles défendent, d'un côté, une ethnomusicologie qui s'appuie plus systématiquement sur les conceptions locales de l'environnement sonore et, de l'autre, une écologie scientifique qui, pour comprendre l'évolution des milieux sonores, prenne mieux en compte les effets de l'activité humaine sur la nature. Dans le quatrième chapitre, Robin Ryan étudie l'impact des changements environnementaux sur la facture de deux instruments de musique aborigènes: le didgeridoo, en bois d'eucalyptus, et la feuille du même arbre, utilisée comme aérophone. Enfin, Jeff Todd Titon (chap. 5) revisite les écrits du philosophe et naturaliste américain Henry David Thoreau. Il examine sa manière, résolument moderne, d'envisager l'écoute comme expérience sensible sans poser a priori de distinction entre sons de l'environnement que ceux-ci émanent de la nature ou soient produits par des animaux ou des hommes et sons musicaux.

5 Cette continuité entre nature et culture, entre sons ambiants et sons organisés, est au cœur des deux premiers chapitres de la seconde partie, "Fieldwork directions". Anthony Seeger (chap. 6) attire ainsi l'attention des écomusicologues sur la notion de 
perspectivisme ${ }^{2}$, les invitant à relativiser, au sein d'un champ encore naissant, la valeur des conceptions occidentales de la nature, de l'homme et de la musique. Il illustre son propos d'un exemple amazonien, dans lequel il montre que la catégorisation des répertoires musicaux des indiens Kĩsêdjê (ou Suyá) ne peut être comprise que si l'on admet, avec eux, qu'ils ne peuvent pas créer de nouvelles mélodies et n'ont d'autre choix que d'entrer en communication avec les espèces animales environnantes - ellesmêmes humaines et percevant les Kĩsêdjê comme une espèce animale - pour apprendre de nouveaux chants. Helena Simonett (chap.7) montre de son côté combien les pratiques musicales et chorégraphiques des Mayo-Yoreme, communauté du Nord-Ouest mexicain, sont au cœur d'un dispositif original où les hommes se transforment en oiseaux pour partager avec eux leurs expériences sensorielles et reformuler, sur l'instant, leur univers sonore. Les chapitres suivants contrastent avec ceux de Seeger et Simonet: l'enquête ethnographique paraît moins centrale et les discours davantage marqués par des préoccupations environnementalistes. Kevin Dawe (chap. 8) s'intéresse ainsi aux difficultés que rencontrent des facteurs de guitares et de luths, espagnols, crétois, écossais et ougandais, pour concilier et harmoniser exigences musicales, éthiques locales et développement durable. Andrew Mark (chap.9) se penche quant à lui sur la manière dont les habitants d'une communauté post-hippie de Colombie britannique utilisent la musique pour résoudre les problèmes démographiques, économiques et environnementaux qu'ils rencontrent. Enfin, Maria Sonevytsky et Adrian Ivakhiv (chap. 10) montrent combien la catastrophe nucléaire de Tchernobyl entraîna non seulement une vague de revendications nationalistes, identitaires et environnementalistes, mais aussi un regain d'intérêt pour les traditions musicales de la région, considérées comme authentiques et emblématiques de la culture villageoise ukrainienne.

6 Les parties trois et quatre regroupent des textes plus fortement marqués par les études critiques, ou critical studies. James Rhys Edwards (chap.11) propose une lecture postmoderne de l'écomusicologie dans laquelle il tente de montrer ses liens avec divers courants théoriques, de l'école de Frankfort à l'écologie politique marxiste contemporaine (p.153). Les chapitres suivants (de 12 à 20), bien que s'inscrivant en grande majorité dans cette perspective critique, mobilisent des ressources fort diverses, empruntant tour à tour aux sound studies (Alexandra Hui, chap. 13 ; et Aaron S. Allen, chap. 20), à la sociologie des médias (Travis D. Stimeling, chap. 14), à l'écocritique (David Ingram, chap. 16 ; et Eric Drott, chap. 17) et aux études de genre (Sabine Feisst, chap. 18 ; et Denise Von Glahn, chap. 19). Dans tous les cas, l'accent est placé en priorité sur les dimensions politiques et éthiques du son. Pour exemple, l'étude de Mark Pedelty (chap. 15) sur l'utilisation (plutôt attendue) de la chanson pour diffuser un point de vue sur la défense de l'environnement; celle de Denise Von Glahn (chap. 19) sur le travail de la compositrice américaine Libby Larsen et ses liens avec l'écoféminisme et le biorégionalisme; ou celle de W. Luke Windsor (chap.12) sur l'enseignement universitaire au Royaume-Uni. Ce dernier, s'appuyant sur les avancées de la psychologie et une approche écologique de la perception héritée des travaux de James J. Gibson ${ }^{3}$, montre que les enseignements musicaux dispensés dans les universités britanniques tendent à véhiculer des oppositions - entre nature et culture, bruit et musique - qui ne correspondent pas à la réalité des pratiques et des faits. Il propose en contrepartie une approche didactique basée sur l'improvisation musicale libre et collective, dans laquelle les élèves, à travers le jeu instrumental et le chant, la créativité 
et l'écoute, dialoguent entre eux, en temps réel, au sein d'une situation et d'un environnement en perpétuel mouvement.

7 L'hétérogénéité des chapitres peut sans doute être considérée comme un atout. L'écomusicologie ne serait-elle pas, selon Allen et Dawe, un champ thématique où tous les croisements disciplinaires seraient possibles? L'argument, pourtant, peine à convaincre totalement. Ainsi, par exemple, le surlignage systématique et appuyé des liens entre les chapitres dans l'introduction générale et les introductions de partie (p. 4-6, 18-22, 82-86, 148-151, 214-217) donne l'impression qu'Allen et Dawe veulent à tout prix justifier leur entreprise et pérenniser un terme, " ecomusicology », dont on peut questionner la nécessité et la valeur heuristique. Par ailleurs, les chapitres ne communiquent guère entre eux au-delà du simple échange thématique, laissant au final peu d'espoir - ce que les auteurs reconnaissent eux-mêmes (p.11) - sur le développement futur d'un paradigme commun et d'une méthodologie un tant soit peu unifiée. Ceci dit, plusieurs textes laissent entrevoir la possibilité d'une construction plus solide et de réels échanges interdisciplinaires. Tel est le cas, notamment, du deuxième chapitre, dans lequel Boyle et Waterman montrent combien l'utilisation conjointe des méthodes d'analyse de l'ethnomusicologie et de l'écologie scientifique nous permettrait de mieux comprendre les interconnections entre performance, musique et contexte. Mais de telles collaborations, parce qu'elles nécessitent des points de rencontre à la fois concrets et précis, semblent plus faciles à mettre en place dans le cadre de disciplines empiriques, où l'observation est la condition sine qua non de la recherche, que dans celui de disciplines critiques, où l'assemblage théorique fait souvent œuvre de méthodologie. Or, dans l'état actuel des choses, l'écomusicologie s'apparente davantage à un projet critique et politique né dans une période de crise environnementale qu'à l'élaboration d'un champ de recherche fondé sur des bases empiriques. Mais si l'objet, les méthodes et les limites de l'écomusicologie restent encore imprécis, celle-ci a néanmoins l'avantage, comme toute entreprise naissante, d'être extrêmement dynamique. Présageons donc que ce premier grand panorama ouvre le débat et permette d'interroger plus avant le caractère hybride et le potentiel opérationnel d'un champ en devenir, à la fois stimulant et passionnant.

\section{NOTES}

1. Voir par exemple: GUY, Nancy, «Flowing down Taiwan's Tamsui River : Towards an Ecomusicology of the Environmental Imagination », Ethnomusicology 53, no 1, 2009, p. 218-248 ; PEDELTY, Mark, Ecomusicology: Rock, Folk, and the Environment, Philadelphie, Temple University Press, 2012; ou TITON, Jeff Todd, "The Nature of Ecomusicology ", Música e Cultura 8, nº 1, 2013, p. 8-18.

2. Voir notamment : VIVEIROS DE CASTRO, Eduardo, «Os pronomes cosmológicos e o perspectivismo ameríndio », Mana 2, no 2, 1996, p. 115-144.

3. Par exemple: GIBSON, James J., The Ecological Approach to Visual Perception, Boston, Houghton Mifflin, 1979. 


\section{AUTEURS}

\section{WILLIAM TALLOTTE}

William Tallotte est ethnomusicologue et travaille depuis près de 20 ans sur les pratiques musicales de l'Inde du Sud. Il a soutenu une thèse de doctorat à l'Université Paris Sorbonne (Paris 4) et enseigné dans plusieurs universités françaises (Bordeaux 3, Strasbourg 2, Rennes 2). Il a été post-doctorant au musée du quai Branly (Paris), puis Eurias Junior Research Fellow au New Europe College (Bucarest) et Marie Curie Intra-European Research Fellow à la School of Oriental and African Studies (Londres). Il poursuit actuellement des recherches sur les musiques rituelles des communautés d'origine indienne et tamoules de l'océan Indien (Mascareignes et Sri Lanka). 